\title{
Reconhecimento dos sinais e sintomas e dos fatores de risco do acidente vascular cerebral por leigos: uma revisão integrativa
}

\author{
Recognition of signs and symptoms and risk factors for stroke by lay people: an integrative review \\ Reconocimiento de signos y síntomas y factores de riesgo de accidente cerebrovascular por \\ personas no profesionales: una revisión integradora
}

Sued Magalhães Moita

ORCID: https://orcid.org/0000-0001-7189-6199 Universidade Estadual do Ceará, Brasil E-mail: sued.moita@aluno.uece.br Andressa Nogueira Cardoso ORCID: https://orcid.org/0000-0002-7332-7560 Nome da instituição onde atua, País E-mail: andressa.nogueira@aluno.uece.br Ingred Pimentel Guimarães ORCID: https://orcid.org/0000-0003-0146-0589 Universidade Estadual do Ceará, Brasil E-mail: ingred.pimentel@aluno.uece.br

Karine Souza Rodrigues

ORCID: https://orcid.org/0000-0002-4229-4006 Universidade Estadual do Ceará, Brasil E-mail: kar.rodrigues@aluno.uece.br Manoelise Linhares Ferreira Gomes ORCID: https://orcid.org/0000-0003-1639-684X Universidade Estadual Vale do Acaraú, Brasil E-mail: manoeliselfg@ gmail.com Vitória Ferreira do Amaral ORCID: https://orcid.org/0000-0003-4255-2033 Universidade Federal do Ceará, Brasil E-mail: vyctoriaamaral@gmail.com

Francisco José Maia Pinto

ORCID: https://orcid.org/0000-0003-2976-7857 Universidade Estadual do Ceará, Brasil E-mail: francisco.pinto@uece.br

Cybelle Façanha Barreto Medeiros Linard ORCID: https://orcid.org/0000-0001-7927-9320 Universidade Estadual do Ceará Brasil E-mail: cybellelinard@yahoo.com.br

\begin{abstract}
Resumo
Objetivo: Investigar o nível de conhecimento dos leigos acerca da identificação dos sinais e sintomas e dos fatores de risco referentes ao AVC. Materiais e métodos: Foi realizada pesquisa bibliográfica em bases de dados internacionais, sendo selecionados 9 estudos publicados entre os anos de 2017 e 2021, compreendendo os idiomas inglês e português. Resultados: Ao analisar os artigos elencados, percebeu-se que a maioria fez uso de questionário como instrumento de coleta de dados $(88,9 \%)$ e entrevistas presenciais $(66,7 \%)$. Com relação aos sinais e sintomas, os principais abordados pelos estudos foram: fraqueza ou formigamento na face, no braço ou na perna; confusão, alteração da fala ou compreensão da linguagem; alteração na visão e dor de cabeça súbita, intensa e sem causa aparente. Quanto aos fatores de risco, os mais abordados foram hipertensão e tabagismo. Conclusões: Percebeu-se que o nível de conhecimento dos leigos acerca da identificação dos sinais e sintomas e fatores de risco do AVC não é satisfatório, devido ao baixo nível educacional dos participantes, poucas campanhas educativas e diminuto vínculo entre os usuários e as equipes interdisciplinares de saúde.
\end{abstract}

Palavras-chave: Sinais e sintomas; Fatores de risco; Acidente vascular cerebral.

\section{Abstract}

Objective: To investigate the level of knowledge of laypeople about the identification of signs and symptoms and risk factors related to stroke. Materials and methods: Bibliographic research was carried out in international databases, and 
9 studies published between the years 2017 and 2021 were selected, comprising the languages English and Portuguese. Results: By analyzing the articles listed, it was noticed that the majority used a questionnaire as an instrument for data collection $(88.9 \%)$ and face-to-face interviews $(66.7 \%)$. With regard to signs and symptoms, the main ones addressed by the studies were: weakness or tingling in the face, arm or leg; confusion, speech disorder or language comprehension; change in vision and sudden, severe headache with no apparent cause. As for risk factors, the most addressed were hypertension and smoking. Conclusions: It was observed that the level of knowledge of laypeople about the identification of signs and symptoms and risk factors for stroke is not satisfactory, due to the low educational level of the participants, few educational campaigns and a small link between users and interdisciplinary teams of health.

Keywords: Signs and symptoms; Risk factors; Stroke.

\section{Resumen}

Objetivo: Investigar el nivel de conocimiento de los profanos sobre la identificación de signos y síntomas y factores de riesgo relacionados con el ictus. Materiales y métodos: Se realizó una búsqueda bibliográfica en bases de datos internacionales y se seleccionaron 9 estudios publicados entre 2017 y 2021, comprendidos en los idiomas inglés y portugués. Resultados: Al analizar los artículos enumerados, se notó que la mayoría utilizó un cuestionario como instrumento de recolección de datos $(88,9 \%)$ y entrevistas presenciales $(66,7 \%)$. En cuanto a los signos y síntomas, los principales abordados por los estudios fueron: debilidad u hormigueo en la cara, brazo o pierna; confusión, alteración del habla o comprensión del lenguaje; cambio en la visión y dolor de cabeza repentino, intenso e inexplicable. En cuanto a los factores de riesgo, los más discutidos fueron la hipertensión y el tabaquismo. Conclusões: Percebeu-se que o nível de conhecimento dos leigos acerca da identificação dos sinais e sintomas e fatores de risco do AVC não é satisfatório, devido ao baixo nível educacional dos participantes, poucas campanhas educativas e diminuto vínculo entre os usuários e as equipes interdisciplinares de salud.

Palabras clave: Signos y síntomas; Factores de riesgo; Ictus.

\section{Introdução}

O Acidente Vascular Cerebral (AVC) é caracterizado por déficit neurológico causado por lesão vascular em uma área cerebral, com manifestações clínicas similares, mas com etiologias variadas: AVC hemorrágico (AVCh), que consiste na hemorragia subaracnoide (HSA) e na hemorragia intraparenquimatosa (HIP); e o AVC isquêmico (AVCi) (Radanovic, 2000). Ademais, o termo Acidente Vascular Encefálico (AVE) surgiu para ampliar o conceito, pois essa doença pode envolver qualquer estrutura encefálica e não somente a parte cerebral (Gagliardi, 2010).

Nesse contexto, os fatores de risco para o AVC são divididos em modificáveis, que são sobretudo a Hipertensão Arterial Sistêmica (HAS), o Diabetes Mellitus (DM) e o tabagismo; e não modificáveis, que são idade avançada, sexo masculino e raça negra (Araújo et al., 2018). A identificação e o controle desses fatores são essenciais, pois permitem a redução significativa da incidência do AVC (Pires et al., 2004).

Os sinais e sintomas ajudam no reconhecimento do AVC, como fraqueza ou formigamento na face, em membros superior e inferior, de modo unilateral; confusão mental; alteração da fala ou compreensão, da visão, do equilíbrio e do andar; tontura e cefaleia súbita, intensa e sem causa aparente (Sociedade Brasileira De Doenças Cerebrovasculares, 2021). Destacase, ainda, que a falta de reconhecimento desses sinais e sintomas gera o atraso na busca por atendimento em saúde, o que ocasiona impactos negativos no tratamento pós AVC e reduz a probabilidade de recuperação (Faria et al., 2017).

O AVC é a segunda maior causa de óbitos no mundo, sendo responsável por cerca de $11 \%$ do total no ano de 2019 (Organização Mundial Da Saúde, 2019). A Organização Mundial da Saúde (OMS) estima que, até 2030, o AVC permaneça com essa colocação (Araújo et al., 2018). Paralelo a isso, no Brasil, o AVC é a doença de maior mortalidade (Sociedade Brasileira De Doenças Cerebrovasculares, 2021) e, apesar da regressão do número de óbitos, o Nordeste permanece com taxas elevadas (Araújo et al., 2018). Isto se comprova através de dados analisados do Sistema de Informações sobre Mortalidade (SIM), no período de 1990 a 2012, que evidenciou, na maioria das regiões do país, redução nas taxas de mortalidade ajustadas para idade, à exceção do Nordeste (Oliveira et al., 2020). 
Ademais, estudo realizado no Brasil em 2004 e 2005, com o objetivo de mensurar a conscientização de leigos sobre o AVE, revelou resultados alarmantes, já que 22\% dos indivíduos disseram não conhecer nenhum sinal indicativo dessa doença, e apenas 34,6\% dos sujeitos responderam o número correto do telefone nacional de emergência (192) (Pontes-Neto et al., 2008). Outra abordagem relevante são os custos para o Sistema Único de Saúde (SUS), haja vista o AVE e a Insuficiência Cardíaca (IC) terem sido responsáveis pelos mais altos valores reembolsados, entre 2008 e 2018, em procedimentos clínicos, acumulando, nesse período, $\mathrm{R}$ 8,4 bilhões em gastos (Oliveira et al., 2020).

Devido a isso, justifica-se a análise desta temática, pois o reconhecimento rápido dos sinais e sintomas possibilita o contato mais célere com os Serviços Médicos de Emergência (SME) e tratamento eficaz. Assim, minimizar o atraso na busca do SME viabiliza reduzir óbitos e custos devido ao AVC. Além disso, a prevenção do transtorno de alta prevalência e amplamente evitável, como é o caso dessa patologia, é obtida com mais frequência quando os fatores de risco são identificados precocemente (Gomes et al., 2017).

Diante do exposto, o objetivo da presente revisão integrativa foi investigar, na literatura científica, o nível do conhecimento dos leigos acerca da identificação dos sinais e sintomas e dos fatores de risco referentes ao Acidente Vascular Cerebral (AVC).

\section{Materiais e Métodos}

O presente estudo consiste em uma revisão integrativa da literatura, método de análise que possibilita a busca, a avaliação e a síntese das informações disponíveis sobre o tema pesquisado. As etapas metodológicas foram: identificação da temática e seleção da questão norteadora; definição dos critérios de inclusão e exclusão; determinação das informações a serem extraídas; análise dos estudos incluídos; interpretação dos resultados e apresentação da revisão (Sousa et al., 2017).

A princípio, o tema estabelecido foi "Reconhecimento dos sinais e sintomas e dos fatores de risco do Acidente Vascular Cerebral por leigos", e a pergunta norteadora foi "Qual o nível de reconhecimento dos leigos acerca dos sinais e sintomas e fatores de risco do Acidente Vascular Cerebral?".

Os critérios de inclusão estabelecidos foram: estudos relacionados com a temática; artigos originais publicados nos últimos cinco anos, em inglês e português; estudos observacionais, transversais, ensaios clínicos e relatos de casos clínicos. Em paralelo, os critérios de exclusão foram: editoriais, short communication, capítulos de livro, estudos de grupo de foco e teses, além de estudos que não especificaram os principais sinais e sintomas e fatores de risco evidenciados pelos partícipes.

A estratégia de busca foi desenvolvida a partir dos seguintes descritores em inglês registrados no Medical Subject Headings (MeSH): "stroke", "signs and symptoms" e "risk factors"; e dos descritores correspondentes em português no Descritores em Ciência da Saúde (DeCS): "acidente vascular cerebral", "sinais e sintomas" e "fatores de risco". O operador booleano "AND" foi utilizado para associar os descritores na busca.

Em seguida, em março de 2021, foi realizada a busca pelos estudos, por intermédio do portal de periódicos da Coordenação de Aperfeiçoamento de Pessoal de Nível Superior (CAPES), nas bases de dados eletrônicas: Medical Literature Analysis and Retrieval System Online (MEDLINE), Literatura Latino-Americana e do Caribe em Ciências da Saúde (LILACS) e Scopus, conforme apresentado na Tabela 1. 
Tabela 1. Estratégia de busca nas bases de dados Medline, Scopus e Lilacs, Brasil, 2021.

\begin{tabular}{|ccc}
\hline $\begin{array}{c}\text { Bases de } \\
\text { dados }\end{array}$ & Estratégia de busca & $\begin{array}{c}\text { Artigos } \\
\text { encontrados }\end{array}$ \\
\hline Medline & Stroke AND "Signs and Symptoms" AND "Risk \\
Factors" & 48 \\
Scopus & Stroke AND "Signs and Symptoms" AND "Risk \\
& $\begin{array}{c}\text { Factors" } \\
\text { Lilacs }\end{array}$ & 60 \\
& Stroke AND "Signs and Symptoms" AND "Risk \\
Factors" & 7 \\
\hline
\end{tabular}

Fonte: Autores.

\section{Resultados}

Após a triagem a partir da leitura de títulos, resumos e análise de acordo com os critérios de inclusão e exclusão, foram selecionados 17 artigos. Desse total, foram excluídas 8 duplicatas, culminando em 9 artigos. A adaptação do instrumento Preferred Reporting Items for Systematic Review and Meta-Analyses (PRISMA) (Moher et al., 2009) foi utilizada para demonstrar esse processo (Figura 1).

Figura 1. Processo de busca e seleção dos artigos.

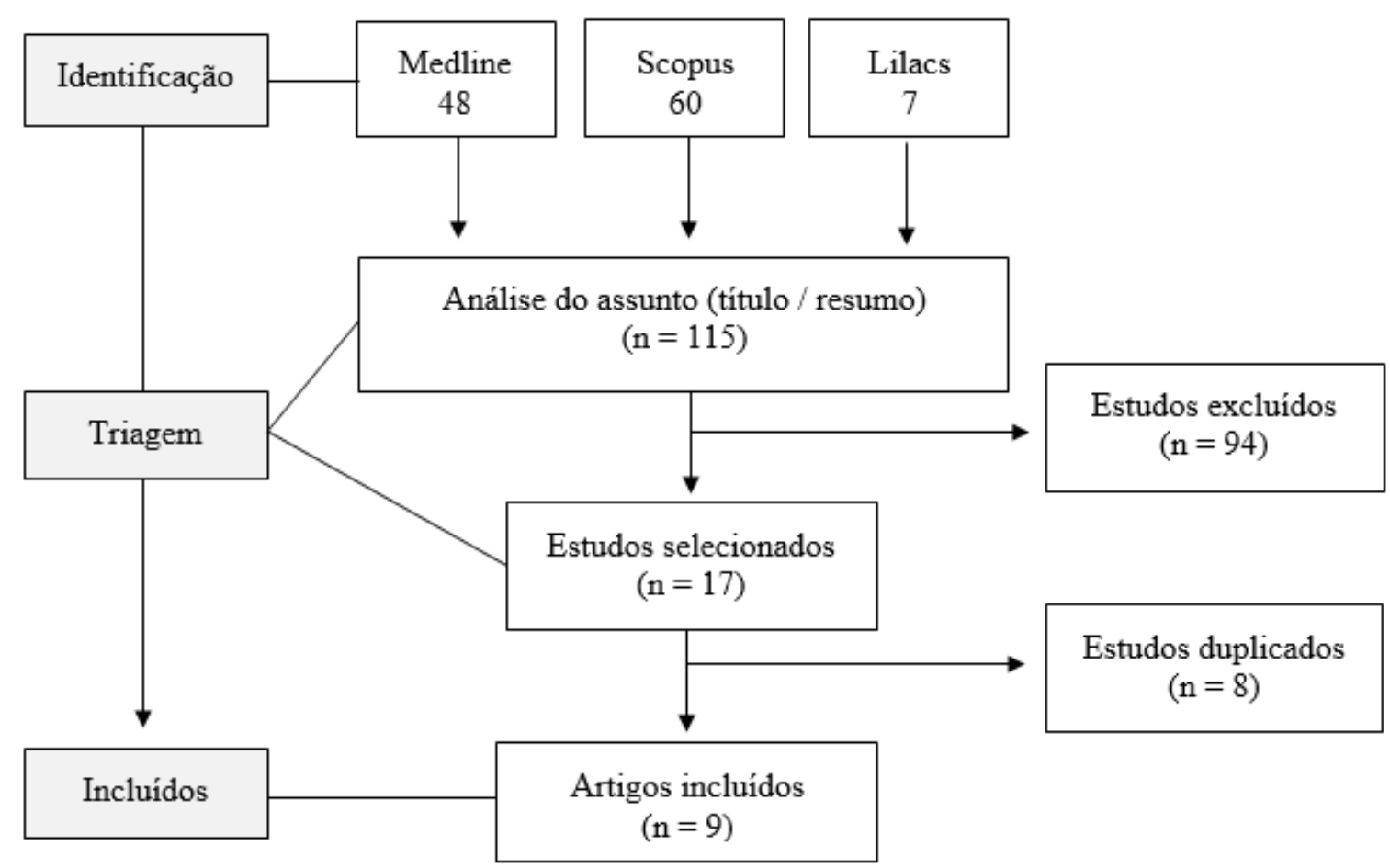

Fonte: Autores. 
Para extração e avaliação dos dados, utilizou-se tabela contendo informações a serem localizadas nos artigos, como: título, ano de publicação, base de dados em que foi encontrado, periódico em que foi publicado, bem como seu respectivo qualis em Saúde Coletiva, local, método de pesquisa, nível de evidência, amostra, variáveis analisadas, objetivos, sinais e sintomas e fatores de risco para o AVC investigados, além de achados que respondessem à pergunta norteadora (Tabela 2).

Enquanto estratégia para interpretação dos resultados, foi consolidada a frequência dos principais sinais e sintomas e fatores de risco do AVC (Tabelas 3 e 4). Deste modo, após análise, as principais evidências estão estruturadas a partir dos seguintes tópicos temáticos: Reconhecimento dos sinais e sintomas do AVC pela população leiga; Reconhecimento dos fatores de risco do AVC pela população leiga e; Percepção do nível de reconhecimento dos sinais e sintomas e dos fatores de risco do AVC pela população leiga.

Ao analisar os nove artigos elencados como elegíveis para compor a presente revisão, percebeu-se que as amostras tinham mediana de 400 partícipes, sendo a maioria do tipo transversal $(88,9 \%)$, com uso de questionário como instrumento de coleta de dados $(88,9 \%)$ e entrevistas presenciais $(66,7 \%)$. Todos os estudos avaliaram os sinais e sintomas e os fatores de risco reconhecíveis pela população leiga (Tabela 2).

Os sinais e sintomas do AVC abordados nos nove artigos elencados na presente revisão integrativa, foram comparados com os citados pela Sociedade Brasileira de Doenças Cerebrovasculares (SBDCV) (Sociedade Brasileira De Doenças Cerebrovasculares, 2021). Observou-se que todos os estudos incluíram os seguintes sinais e sintomas: fraqueza ou formigamento na face, no braço ou na perna; confusão, alteração da fala ou compreensão da linguagem; alteração na visão (em um ou ambos os olhos) e dor de cabeça súbita, intensa e sem causa aparente. Paralelo a isso, a alteração do equilíbrio, coordenação, tontura ou alteração no andar foi identificada em sete estudos (77,8\%) (Tabela 3). 
Tabela 2. Coleta geral de informações dos artigos selecionados, Brasil, 2021.

\begin{tabular}{|c|c|c|c|c|c|c|c|}
\hline $\begin{array}{l}\text { Codificação } \\
\text { dos artigos }\end{array}$ & Referência & Amostra & $\begin{array}{l}\text { Tipo de } \\
\text { estudo }\end{array}$ & $\begin{array}{l}\text { Coleta de } \\
\text { dados }\end{array}$ & $\begin{array}{c}\text { Tipo de } \\
\text { entrevista }\end{array}$ & Local & $\begin{array}{c}\text { Conhecimentos } \\
\text { avaliados }\end{array}$ \\
\hline A & $\begin{array}{l}\text { Hosseininezhad et al. } \\
\qquad \text { (2017) }\end{array}$ & $\mathrm{n}=649$ & Transversal & Questionário & Por telefone & Irã & SS, FR, FI \\
\hline $\mathrm{B}$ & Nansseu et al. (2017) & $\mathrm{n}=1025$ & Transversal & Questionário & Presencial & Camarões & DF, SS, FR, CQ \\
\hline $\mathrm{C}$ & $\begin{array}{l}\text { Góngora-Riveraa et al. } \\
\qquad(2018)\end{array}$ & $\mathrm{n}=3632$ & $\begin{array}{l}\text { Transversal, } \\
\text { observacional } \\
\text { e analítico }\end{array}$ & Questionário & Presencial & México & $\mathrm{SS}, \mathrm{FR}, \mathrm{CD}, \mathrm{CQ}$ \\
\hline $\mathrm{D}$ & $\begin{array}{l}\text { Osahon \& Ekanem. } \\
\qquad(2018)\end{array}$ & $\mathrm{n}=238$ & Prospectivo & Questionário & Presencial & Nigéria & $\begin{array}{c}\mathrm{DF}, \mathrm{SS}, \mathrm{FR}, \mathrm{PV}, \\
\mathrm{TT}\end{array}$ \\
\hline $\mathrm{E}$ & Chhabra et al. (2019) & $\mathrm{n}=350$ & Transversal & Questionário & Por telefone & Índia & SS, FR, TT \\
\hline $\mathrm{F}$ & $\begin{array}{l}\text { Krishnamurthi et al. } \\
\qquad(2020)\end{array}$ & $\mathrm{n}=400$ & Transversal & Questionário & Por telefone & $\begin{array}{l}\text { Nova } \\
\text { Zelândia }\end{array}$ & $\mathrm{SS}, \mathrm{FR}, \mathrm{CD}, \mathrm{FI}$ \\
\hline $\mathrm{G}$ & Machado et al. (2020) & $\mathrm{n}=375$ & Transversal & Questionário & Presencial & Brasil & SS, FR \\
\hline
\end{tabular}


Research, Society and Development, v. 10, n. 10, e587101019340, 2021

(CC BY 4.0) | ISSN 2525-3409 | DOI: http://dx.doi.org/10.33448/rsd-v10i10.19340

\begin{tabular}{|c|c|c|c|c|c|c|c|}
\hline $\mathrm{H}$ & $\begin{array}{l}\text { Soto-Cámara et al. } \\
\qquad(2020)\end{array}$ & $\mathrm{n}=529$ & $\begin{array}{l}\text { Transversal } \\
\text { prospectivo }\end{array}$ & Questionário & Presencial & Espanha & SS, FR \\
\hline I & $\begin{array}{c}\text { Yesilbalkan et al. } \\
\text { (2019) }\end{array}$ & $n=203$ & $\begin{array}{l}\text { Transversal } \\
\text { comparativo }\end{array}$ & Formulário & Presencial & Turquia & SS, FR \\
\hline
\end{tabular}

Chamadas: FR: fatores de risco; SS: sinais e sintomas; CD: condutas; CQ: consequências ou complicações do AVC; DF: definição; PV: prevenção; TT: tratamentos; FI: fontes de informação sobre o AVC. Fonte: Autores. 
Tabela 3. Frequência dos principais sinais e sintomas do Acidente Vascular Cerebral (AVC) abordados pelos estudos incluídos em relação aos citados pela Sociedade Brasileira de Doenças Cerebrovasculares (SBDCV), Brasil, 2021.

\begin{tabular}{c|c|c}
\hline $\begin{array}{c}\text { Sinais e sintomas para o AVC } \\
\text { (SBDCV) }\end{array}$ & $\begin{array}{c}\text { Estudos que utilizaram esse } \\
\text { sinal / sintoma* }\end{array}$ & $\%$ \\
\hline $\begin{array}{c}\text { Fraqueza ou formigamento na } \\
\text { face, no braço ou na perna }\end{array}$ & A, B, C, D, E, F, G, H, I & 100 \\
$\begin{array}{c}\text { Confusão, alteração da fala ou } \\
\text { compreensão da linguagem }\end{array}$ & A, B, C, D, E, F, G, H, I & 100 \\
$\begin{array}{c}\text { Alteração na visão (em um ou } \\
\text { ambos os olhos) }\end{array}$ & A, B, C, D, E, F, G, H, I & 100 \\
$\begin{array}{c}\text { Dor de cabeça súbita, intensa e } \\
\text { sem causa aparente }\end{array}$ & A, B, C, D, E, F, G, H, I & 100 \\
Alteração do equilíbrio, & & 77,8 \\
andar & A, C, D, E, G, H, I & \\
\hline
\end{tabular}

(*) Os artigos foram codificados em letras. Fonte: Autores.

Outrossim, ao analisar os fatores de risco do AVC abordados nos artigos elencados para esta revisão e compará-los com os citados pela SBDCV (Sociedade Brasileira De Doenças Cerebrovasculares, 2021), observou-se que a hipertensão arterial e o tabagismo foram citados em todos os estudos; uso de álcool e drogas também tiveram alto índice de relato $(88,9 \%)$. Em contrapartida, os dois fatores de risco menos citados nos estudos foram história de doença vascular prévia $(33,3 \%)$ e uso de anticoncepcional $(11,1 \%)$ (Tabela 4$)$. 
Tabela 4. Frequência dos principais fatores de risco do Acidente Vascular Cerebral (AVC) abordados pelos estudos incluídos em relação aos citados pela Sociedade Brasileira de Doenças Cerebrovasculares (SBDCV), Brasil, 2021.

\begin{tabular}{c|c|c}
\hline $\begin{array}{c}\text { Fatores de risco para o AVC } \\
\text { (SBDCV) }\end{array}$ & $\begin{array}{c}\text { Estudos que utilizaram esse } \\
\text { fator de risco* }\end{array}$ & $\%$ \\
\hline tabagismo & A, B, C, D, E, F, G, H, I & 100 \\
álcool e drogas & A, B, C, D, E, F, G, H, I & 100 \\
dislipidemia & A, B, C, D, E, G, H, I & 88,9 \\
obesidade & A, B, C, F, G, H, I & 77,8 \\
diabetes & A, B, C, D, G, H, I & 77,8 \\
idade e sexo & A, C, D, E, G, H, I & 77,8 \\
A, C, D, G, H, I & 66,7 \\
doenças do coração & A, B, C, G, H, I & 66,7 \\
sedentarismo & B, C, F, H, I & 53,6 \\
& A, C, H & 11,1 \\
história de doença vascular prévia & A & 33 \\
\hline
\end{tabular}

(*) Os artigos foram codificados em letras. Fonte: Autores.

\section{Discussão}

\section{Reconhecimento dos sinais e sintomas do AVC pela população leiga}

Considerando o AVC uma doença que apresenta alto índice de mortalidade e elevado potencial incapacitante, o reconhecimento dos sinais e sintomas do AVC pode representar sobrevida e melhor prognóstico (Maniva et al., 2018). Nesse contexto, a Sociedade Brasileira de Doenças Cerebrovasculares (SBDCV) elenca sinais de alerta essenciais para detecção precoce, dos quais a “alteração do equilíbrio, coordenação, tontura ou alteração no andar" foi o único determinante não incluído de forma unânime. Apesar disso, este foi um dos sinais e sintomas mais reconhecidos pela população (64,4\%) em estudo realizado no Irã (Hosseininezhad et al., 2017). Sendo assim, há maior necessidade de atenção a essa manifestação clínica, pois ainda que uma instituição de referência a considere essencial para identificação do AVC, a mesma não foi evidenciada em todos os estudos avaliados.

"Distúrbios da fala" esteve entre os sinais e sintomas mais reconhecidos pela população leiga (Nansseu et al., 2017; Gongora-Rivera et al., 2018; Chhabra et al., 2019; Krishnamurthi et al., 2020). Paralelo a isso, “dor de cabeça súbita, intensa e sem causa aparente", foi evidenciado como variável universal, mas não foi identificada de forma satisfatória por leigos, a exemplo da pesquisa realizada na Índia, em que foi reconhecida pela minoria dos entrevistados $(1,1 \%)(\mathrm{Chhabra}$ et al., 2019). 
Deste modo, independente do destaque atribuído a um determinado sinal e sintoma pelas referências elencadas, isso não implicou relação direta com o nível de conhecimento.

A "fraqueza" foi evidenciada na maioria das pesquisas como um dos principais sinais e sintomas do AVC identificados pela população (Nansseu et al., 2017; Osahon \& Ekanem, 2018; Yesilbalkan et al., 2019; Krishnamurthi et al., 2020; SotoCámara et al., 2020). Entretanto, pesquisa conduzida na Índia (Chhabra et al., 2019) constatou que somente uma pequena parcela dos participantes $(5,7 \%)$ conseguiu identificar tal sinal de alerta. Dessa maneira, percebe-se nível de conhecimento adequado sobre esse fator, devido à prevalência na sua identificação pela população em detrimento de achados atípicos, como no estudo indiano.

Ademais, ao analisar a relação do nível de instrução com o reconhecimento dos sinais e sintomas do AVC, notabilizou-se que sujeitos com maior escolaridade identificaram melhor os sinais e sintomas (Hosseininezhad et al., 2017; Nansseu et al., 2017; Krishnamurthi et al., 2020; Soto-Cámara et al., 2020; Machado et al., 2020). Todavia, em estudo realizado na Turquia (Yesilbalkan et al., 2019), não foi identificada relação entre o nível educacional e o conhecimento sobre os sinais e sintomas do AVC. Destarte, mesmo que não tenha ocorrido consenso entre as pesquisas, percebe-se impactos positivos entre o nível escolar e o melhor reconhecimento do derrame cerebral.

\section{Reconhecimento dos fatores de risco do AVC pela população leiga}

Condições associadas ao aumento do risco em desenvolver uma doença são definidas como fatores de risco (Instituto Nacional Do Câncer, 2021). Estes são divididos em modificáveis, que correspondem à idade, sexo, raça, localização geográfica e hereditariedade; e não modificáveis, que são hipertensão, fibrilação atrial, diabetes mellitus, dislipidemia, obesidade e tabagismo (Sousa Rodrigues et al., 2017). Nessa ótica, a HAS é o principal fator de risco modificável para o AVC e está relacionada à maioria dos casos da doença (80\%) (Gagliardi, 2009). Este fator foi um dos mais reconhecidos pela população, com exceção de estudo (Gongora-Rivera et al., 2018) realizado no México, no qual foi o segundo (23,9\%), atrás apenas do sedentarismo (65,8\%). Dessa maneira, o reconhecimento da hipertensão como fator de risco de AVC foi considerado adequado, o que pode impactar positivamente na prevenção desta doença.

Outrossim, a literatura relata correlação entre o número de cigarros fumados diariamente e maior susceptibilidade ao AVC isquêmico, entre homens abaixo de 50 anos, além de reportar que fumar menos cigarros pode reduzir o risco deste tipo de derrame (Markidan et al., 2018). Nesse contexto, estudos desenvolvidos em Camarões e na Nova Zelândia (Nansseu et al., 2017; Krishnamurthi et al., 2020) evidenciaram o tabagismo como fator de risco mais identificado pela população. Porém, estudo (Chhabra et al., 2019) realizado na Índia inferiu que o tabagismo foi reconhecido apenas por uma pequena parcela dos partícipes $(22,28 \%)$. Ou seja, ainda há déficits na percepção da população de que maus hábitos fumantes geram maior susceptibilidade à ocorrência de derrames, o que pode ser um fator agravante para a incidência de AVC.

O consumo excessivo de bebidas alcoólicas leva à HAS e a níveis alterados de colesterol (Sociedade Brasileira De Doenças Cerebrovasculares, 2021). Nessa conjuntura, estudo (Osahon \& Ekanem, 2018) realizado na Nigéria averiguou que o consumo desregrado de álcool foi um dos fatores de risco mais identificados pela população $(44,1 \%)$. Em contrapartida, estudo (Soto-Cámara et al., 2020) desenvolvido na Espanha revelou que menos da metade dos pacientes apresentaram consumo excessivo de álcool, e somente 3\% reconheceram tal hábito como fator de risco para o AVC. À vista disso, a falta de consenso entre os achados mostra que o consumo desequilibrado de álcool representa desafio, sendo imprescindíveis campanhas informativas com o objetivo de conscientizar, especialmente, o público etilista, o que pode diminuir o consumo dessa droga e, consequentemente, de casos de AVC.

$\mathrm{O}$ estrogênio, presente em anticoncepcionais combinados, estimula transformações hormonais que propiciam o desenvolvimento de AVC (Lima et al., 2017). Nesse sentido, de acordo com a SBDCV, o uso de anticoncepcionais configura-se 
fator de risco importante para o derrame, no entanto foi abordado em apenas uma das referências elencadas, na qual foi reconhecido por diminuto quantitativo de participantes (12,8\%) (Hosseininezhad et al., 2017), o que revela baixa identificação deste fator de risco, ao passo que são insuficientes os estudos que relacionam o nível de conhecimento da população sobre o AVC e o uso de anticoncepcionais.

\section{Percepção do nível de reconhecimento dos sinais e sintomas e dos fatores de risco do AVC pela população leiga}

A capacidade de reconhecimento dos sinais e sintomas e dos fatores de risco do AVC pelo público leigo foi considerada baixa na maioria dos estudos, o que influenciou negativamente a conduta diante de eventos emergenciais, segundo pesquisas realizadas na Índia e na Espanha (Osahon \& Ekanem, 2018; Chhabra et al., 2019; Yesilbalkan et al., 2019; SotoCámara et al., 2020; Machado et al., 2020). Com o intuito de reverter esse quadro, há necessidade de maior atenção à temática, haja vista que a demora na busca por Serviços Médicos de Emergência (SME) pode estar relacionada com o baixo nível de conhecimento de leigos sobre esses determinantes.

Em contrapartida, estudos (Hosseininezhad et al., 2017; Nansseu et al., 2017) realizados em Camarões e no Irã evidenciaram nível satisfatório de reconhecimento da população acerca dos sinais e sintomas e dos fatores de risco para o AVC. Contudo, o questionário estruturado do estudo iraniano, pode ter gerado viés no quantitativo de respostas corretas. Isto porque, em pesquisa (Krishnamurthi et al., 2020) realizada na Nova Zelândia, identificou-se que a consciência da comunidade sobre os principais sintomas e fatores de risco do derrame foi mais alta quando as perguntas eram estruturadas, ao passo que resultados menos favoráveis foram observados em perguntas subjetivas. Desta forma, pode haver relação entre o nível de conhecimento sobre o AVC pela população leiga e a forma de condução da coleta de dados nas pesquisas, uma vez que perguntas estruturadas influenciaram no padrão de resposta dos participantes, o que pode interferir na real consciência sobre a doença.

Estudo (Gongora-Rivera et al., 2018) realizado no México comparou o nível de reconhecimento dos sinais e sintomas e dos fatores de risco do AVC entre as populações rural e urbana. Verificou-se, na primeira, maior conhecimento sobre essa temática, justificada pela melhor relação médico-paciente. Todavia, pesquisa desenvolvida na Nigéria, no ano de 2018, evidenciou nível de conhecimento desanimador sobre os sinais e sintomas pela população rural, o que, segundo os autores, poderia ser decorrente do acesso restrito à informação e à educação (Chukwudelunzu et al., 2018). Notabiliza-se, assim, o importante papel dos profissionais de saúde como disseminadores de informações relativas à prevenção de doenças, sobretudo nas comunidades rurais, o que favorece o autocuidado oportunizado mediante literacia em saúde.

Como contribuições, este estudo proporcionou consolidar, a partir de evidências já publicadas, o reconhecimento dos sinais e sintomas e dos fatores de risco do AVC por leigos.

Dentre as limitações, destaca-se a falta de descrição dos sinais e sintomas e fatores de risco do AVC em três estudos. Embora essas pesquisas respondessem à pergunta norteadora, não foi possível extrair achados mais específicos e de impacto científico para esta revisão. Além disso, devido à inclusão apenas de estudos publicados em inglês e português, evidências relevantes disponíveis em outras línguas poderão ter sido excluídas.

\section{Conclusão}

Conclui-se que o nível de conhecimento dos leigos acerca da identificação dos sinais e sintomas e fatores de risco do AVC não é satisfatório, devido ao baixo nível educacional dos partícipes, diminuto quantitativo de campanhas educativas e inexpressivo vínculo entre os usuários e as equipes interdisciplinares de saúde, o que pressupõe necessidade de serem realizadas mais intervenções educativas em saúde, com o intuito de reverter esse cenário.

Diante da magnitude da temática, sugere-se a realização de novos estudos com amostras maiores e mais diversificadas, tendo como instrumentos de coleta de dados questionários não-estruturados, a fim de inferir o reconhecimento sobre a temática, 
pela esfera populacional inserida nas comunidades rural e urbana.

\section{Agradecimentos}

Os autores agradecem à Liga de Emergência da UECE (LEMERG) por possibilitar a realização dessa obra.

\section{Referências}

Araújo, J. P. D., Darcis, J. V. V., Tomas, A. C. V., \& Mello, W. A. D. (2018). Mortality trend due to cerebrovascular accident in the city of Maringá, Paraná between the years of 2005 to 2015. International Journal of Cardiovascular Sciences, 31, 56-62. doi:10.5935/2359-4802.20170097.

Chhabra, M., Gudi, S. K., Rashid, M., Sharma, P., Sharma, S., \& Khan, H. (2019). Assessment of knowledge on risk factors, warning signs, and early treatment approaches of stroke among community adults in North India: A telephone interview survey. Journal of neurosciences in rural practice, 10(03), 417-422. doi:10.1055/s-0039-1697561.

Chukwudelunzu, F. E., Okwu-Delunzu, V. U., \& Dzissah, J. (2018). Knowledge of stroke signs and symptoms among residents in a rural south eastern Nigerian community. Journal of Stroke and Cerebrovascular Diseases, 27(11), 3306-3310. https://doi.org/10.1016/j.jstrokecerebrovasdis.2018.07.038.

Faria, A. D. C. A., Martins, M. M. F. P. D. S., Schoeller, S. D., \& Matos, L. O. D. (2017). Care path of person with stroke: from onset to rehabilitation. Revista brasileira de enfermagem, 70, 495-503. http://dx.doi.org/10.1590/0034-7167-2016-0579.

Gagliardi, R. J. (2010). Acidente vascular cerebral ou acidente vascular encefálico? Qual a melhor nomenclatura?. Revista Neurociências, 18(2), 131-132. doi: 10.4181/RNC.2010.1802.02p.

Gagliardi, R. J. (2009). Hipertensão arterial e AVC. ComCiência, (109). http://comciencia.scielo.br/scielo.php?script=sci_arttext\&pid=S1519$76542009000500018 \& \operatorname{lng}=$ en $\& n r m=$ is.

Gomes, A. B. A. G. R., Henrique Jr, M., Schoeps, V. A., Santos, M. M. S. A., Pellegrinelli, A., de Matos, B. P., Kubota, G. T., Araújo, H. A., da Silva, L. S. C., Battisti, F. P. L., Kubota, B. Y., Ferreira, A. C., Pellegrino, M. P., Prado, R. A., Abrahm, R., Gagliardi, V. D. B., Simis, M, \& Gagliardi, R. J. (2017). Popular stroke knowledge in Brazil: A multicenter survey during "World Stroke Day". eNeurologicalSci,6, 63-67. https://doi.org/10.1016/j.ensci.2016.12.002.

Gongora-Rivera, F., Gonzalez-Aquines, A., Muruet, W., Barrera-Barrera, S., Leal-Bailey, H., Espinosa-Ortega, M. A., Treviño, A. P., Jacobo-Saucedo, L. A., Villarreal-Velazquez, H. J., Garcia-Ortiz, W., Saucedo-Ostos, Y. Y., Cordero-Perez, A. C., \& Chavez-Luevanos, B. E. (2018). Difference in stroke knowledge between rural and urban communities in a developing country after community-based stroke educational campaigns: results from a cross-sectional study. Neuroepidemiology, 51(3-4), 224-229. https://doi.org/10.1159/000490724.

Hosseininezhad, M., Ebrahimi, H., Seyedsaadat, S. M., Bakhshayesh, B., Asadi, M., \& Ghayeghran, A. R. (2017). Awareness toward stroke in a populationbased sample of Iranian adults. Iranian journal of neurology, 16(1), 7. https://www.ncbi.nlm.nih.gov/pmc/articles/PMC5506760/pdf/IJNL-16-7.pdf.

Instituto Nacional Do Câncer. Ministério Da Saúde. (2021). Prevenção e fatores de risco. https://www.inca.gov.br/causas-e-prevencao/prevencao-e-fatoresde-risco\#: :text=O\%20termo\%20\%22risco\%22\%20\%C3\%A9\%20usado,s\%C3\%A3o\%20chamados\%20fatores $\% 20$ de $\% 20$ risco.

Krishnamurthi, R. V., Barker-Collo, S., Barber, P. A., Tippett, L. J., Dalrymple-Alford, J. C., Tunnage, B., Mahon, S., Parmar, P. G., Moylan, M., \& Feigin, V. L. (2020). Community knowledge and awareness of stroke in New Zealand. Journal of Stroke and Cerebrovascular Diseases, $29(3)$, 104589. https://doi.org/10.1016/j.jstrokecerebrovasdis.2019.104589.

Lima, A. C. S., Martins, L. C. G., Lopes, M. V. D. O., Araújo, T. L. D., Lima, F. E. T., Aquino, P. D. S., \& Moura, E. R. F. (2017). Influence of hormonal contraceptives and the occurrence of stroke: integrative review. Revista brasileira de enfermagem, 70, 647-655. doi: http://dx.doi.org/10.1590/0034-71672016-0056.

Machado, V. S., Hahn, L. D. M., Martins, M. I. M., \& Marrone, L. C. P. (2020). Conhecimento da população sobre acidente vascular cerebral em Torres RS. Rev. bras. neurol, 11-14. https://docs.bvsalud.org/biblioref/2020/09/1120376/rbn_563-versao-final-11-14.pdf.

Maniva, S. J. C. D. F., Carvalho, Z. M. D. F., Gomes, R. K. G., Carvalho, R. E. F. L. D., Ximenes, L. B., \& Freitas, C. H. A. D. (2018). Educational technologies for health education on stroke: an integrative review. Revista brasileira de enfermagem, 71, 1724-1731. https://doi.org/10.1590/0034-7167-20170041 .

Markidan, J., Cole, J. W., Cronin, C. A., Merino, J. G., Phipps, M. S., Wozniak, M. A., \& Kittner, S. J. (2018). Smoking and risk of ischemic stroke in young men. Stroke, 49(5), 1276-1278. https://doi.org/10.1161/STROKEAHA.117.018859.

Moher, D., Liberati, A., Tetzlaff, J., Altman, D. G., \& Prisma Group. (2009). Preferred reporting items for systematic reviews and meta-analyses: the PRISMA statement. PLoS medicine, 6(7), e1000097. https://doi.org/10.1371/journal.pmed.1000097.

Nansseu, J. R., Atangana, C. P., Petnga, S. J. N., Kamtchum-Tatuene, J., \& Noubiap, J. J. (2017). Assessment of the general public's knowledge of stroke: a cross-sectional study in Yaoundé, Cameroon. Journal of the neurological sciences, 378, 123-129. https://doi.org/10.1016/j.jns.2017.05.004.

Oliveira, G. M. M. D., Brant, L. C. C., Polanczyk, C. A., Biolo, A., Nascimento, B. R., Malta, D. C., de Souza, M. F. M., Soares, G. P., Junior, G. F. X., Machline-Carrion, M. J., Bittencourt, M. S., Neto, O. M. P., Silvestre, O. M., Teixeira, R. A., Sampaio, R. O., Gaziano, T. A., Roth, G. A., \& Ribeiro, A. L. P. (2020). Cardiovascular Statistics-Brazil 2020. Arquivos Brasileiros de Cardiologia, 115, 308-439. https://doi.org/10.36660/abc.20200812.

Organização Mundial Da Saúde. (2019). The top 10 causes of death. https://www.who.int/news-room/fact-sheets/detail/the-top-10-causes-ofdeath\#: :text=The\%20top $\% 20$ global\%20causes $\% 20$ of,birth $\% 20$ asphyxia\%20and\%20birth\%20trauma\%2C. 
Research, Society and Development, v. 10, n. 10, e587101019340, 2021

(CC BY 4.0) | ISSN 2525-3409 | DOI: http://dx.doi.org/10.33448/rsd-v10i10.19340

Osahon, P. T., \& Ekanem, E. N. (2018). Assessment of Stroke Awareness Among Public Servants in a Study Population in Nigeria. Tropical Journal of Natural Product Research, 2(6), 278-281. https://doi.org//10.26538/tjnpr/v2i6.4.

Pires, S. L., Gagliardi, R. J., \& Gorzoni, M. L. (2004). Estudo das freqüências dos principais fatores de risco para acidente vascular cerebral isquêmico em idosos. Arquivos de Neuro-Psiquiatria, 62, 844-851. https://doi.org/10.1590/S0004-282X2004000500020.

Pontes-Neto, O. M., Silva, G. S., Feitosa, M. R., De Figueiredo, N. L., Fiorot Jr, J. A., Rocha, T. N., Massaro, A. R., \& Leite, J. P. (2008). Stroke awareness in Brazil: alarming results in a community-based study. Stroke, 39(2), 292-296. https://doi.org/10.1161/STROKEAHA.107.493908.

Radanovic, M. (2000). Características do atendimento de pacientes com acidente vascular cerebral em hospital secundário. Arquivos de NeuroPsiquiatria, 58, 99-106. https://doi.org/10.1590/S0004-282X2000000100015.

Sociedade Brasileira de Doenças Cerebrovasculares. (2021). Acidente http://www.sbdcv.org.br/publica_avc.asp\#: :text=Fraqueza\%20ou\%20formigamento\%20na\%20face,tontura\%20ou\%20altera\%C3\%A7\%C3\%A3o\%20no\%2 Oandar.

Soto-Cámara, R., González-Bernal, J. J., González-Santos, J., Aguilar-Parra, J. M., Trigueros, R., \& López-Liria, R. (2020). Knowledge on signs and risk factors in stroke patients. Journal of clinical medicine, 9(8), 2557. https://doi.org/10.3390/jcm9082557.

Sousa, L. M. M., Marques-Vieira, C. M. A., Severino, S. S. P., \& Antunes, A. V. (2017). A metodologia de revisão integrativa da literatura em enfermagem. $N^{o} 21$ Série 2-Novembro 2017, 17. http://www.sinaisvitais.pt/images/stories/Rie/RIE21.pdf\#page=17.

Sousa Rodrigues, M., Fernandes, L., \& Galvao, I. M. (2017). Modifiable and non-modifiable risk factors for ischemic stroke: a descriptive approach/Fatores de risco modificaveis e nao modificaveis do AVC isquemico: uma abordagem descritiva. Revista de Medicina, 96(3), 187-193. https://www.revistas.usp.br/revistadc/article/download/123442/133973/269007.

Yesilbalkan, O. U., Karadakovan, A., Dogru, B. V., Akman, P., Ozel, E., \& Bozturk, Y. (2019). Awareness of risk factors and warning signs of stroke among caregivers of patient with and not with stroke: results from questionnaire. J Pak Med Assoc, 69(8), 1114-1118. https://www.jpma.org.pk/PdfDownload/9272. 